\title{
Extracellular glutamate and GABA transients at the transition from interictal spiking to seizures
}

Yoshiteru Shimoda*1, Vincent Magloire ${ }^{* 1 \#}$, Jonathan S Marvin², Marco Leite ${ }^{1}$, Loren L Looger $^{2}$, Dimitri M Kullmann" ${ }^{1 \#}$

1. UCL Institute of Neurology, Queen Square, WC1N3BG, London, UK.

2. Janelia Research Campus, Howard Hughes Medical Institute, Ashburn, VA, USA

*These authors contributed equally.

\# Corresponding authors: v.magloire@ucl.ac.uk and d.kullmann@ucl.ac.uk

Running title: Impaired GABA release at seizure onset

Number of pages: 31

Number of figures: 7 plus 5 supplementary figures

Number of words: $\quad$ Abstracts: 137

Whole manuscript: 8162

\section{Keywords: GABA, Glutamate, Seizures, Imaging}

Acknowledgements: We are grateful to members of the Experimental Epilepsy Group at the UCL Queen Square Institute of Neurology for advice and technical assistance. This work was supported by Epilepsy Research UK, the Medical Research Council and The Wellcome Trust. V.M. is the recipient of an Emerging Leader Fellowship from Epilepsy Research UK (F1901). Y.S. received funding from the Japan Epilepsy Research Foundation.

Author contributions: Y.S., V.M., D.M.K. conceived the experiments. Y.S and V.M. performed the experiments. Y.S., V.M. and M.L. analysed the data. J.S.M and L.L.L. provided the iGABASnFR constructs. V.M, Y.S. and D.M.K wrote the manuscript and all the authors provided feedbacks. D.M.K. secured funding. 


\section{Abstract}

Focal epilepsy is associated with intermittent brief population discharges (interictal spikes), which resemble sentinel spikes that often occur at the onset of seizures. Why interictal spikes self-terminate whilst seizures persist and propagate is incompletely understood. Here we use fluorescent glutamate and GABA sensors in an awake rodent model of neocortical seizures to resolve the spatiotemporal evolution of both neurotransmitters in the extracellular space. Interictal spikes are accompanied by brief glutamate transients which are maximal at the initiation site and rapidly propagate centrifugally. GABA transients last longer than glutamate transients and are maximal $\sim 1.5 \mathrm{~mm}$ from the focus where they propagate centripetally. At the transition to seizures, GABA transients are attenuated, whilst glutamate transients increase in spatial extent. The data imply that an annulus of feed-forward GABA release intermittently collapses, allowing seizures to escape from local inhibitory restraint. 


\section{Introduction}

Epilepsy is characterized by a propensity for seizures, which typically only occur intermittently although can have devastating consequences for the patient if they are allowed to persist. Many types of seizures are recognized, with various clinical manifestations, electro-encephalographic (EEG) correlates and etiologies. Nevertheless, a very common pattern is for seizures to arise from a specific cortical region, and to spread to involve local networks or to generalize to both hemispheres. Such focal or partial-onset epilepsy is very often accompanied by interictal discharges (IIDs), which can be used clinically to help localize the seizure onset zone (de Curtis and Avanzini, 2001). IIDs (also known as interictal spikes) are electrographically similar to large-amplitude discharges that often occur at the start of a seizure (so-called sentinel spikes (Lévesque et al., 2018)). The mechanisms underlying interictal spikes are incompletely understood, as are the reasons why spikes can sometimes proceed to seizures. Interictal spikes are dominated by GABAergic signaling, consistent with powerful feed-forward inhibition confining the spatial and temporal extent of hypersynchronous firing of principal neurons (Bohannon and Hablitz, 2017; Huberfeld et al., 2011; Muldoon et al., 2015; Trevelyan et al., 2006). Collapse of this inhibitory restraint provides a parsimonious explanation why seizures occur intermittently. This principle is supported by in vitro models, as well as in vivo electrical recordings in both experimental models and patients with refractory epilepsy (Liou et al., 2018; Muldoon et al., 2015; Parrish et al., 2019; Schevon et al., 2012; Trevelyan et al., 2006, 2007). It is also consistent with the spatiotemporal evolution of neuronal activity measured using fluorescent calcium indicators in awake head-fixed rodents, where interictal spikes and seizures are initially indistinguishable (Rossi et al., 2017).

How feed-forward inhibition collapses remains unclear. Several possible mechanisms have been proposed. These include chloride accumulation in principal neurons upon intense activation of $\mathrm{GABA}_{A}$ receptors, which could in turn attenuate the inhibitory effect of these receptors and even promote GABA-mediated depolarization (Cohen, 2002; Pavlov et al., 2013), facilitating the recruitment of principal neurons. Chloride accumulation could also lead to extracellular potassium accumulation secondary to activation of the chloride exporter KCC2 (Avoli et al., 1996; Librizzi et al., 2017; Viitanen et al., 2010). Alternatively, collapse of an inhibitory restraint could occur as a result of a failure of GABA release, for instance because of vesicle depletion, presynaptic inhibition of GABAergic terminals (Lei and McBain, 2003; Zhang et al., 2012), or because intense depolarization of interneurons prevents them from firing (Cammarota et al., 2013; Karlócai et al., 2014). Evidence has been put forward in support of all of the above hypotheses, and they are not mutually exclusive, especially when 
the diversity of interneurons is taken into account (Magloire et al., 2018). However, much of the available evidence comes from highly reduced preparations of debatable relevance to seizure mechanisms in vivo. Furthermore, experimental manipulations that interfere with different aspects of inhibitory signaling to investigate how inhibition collapses are themselves pro-epileptic.

Imaging epileptiform activity in awake head-fixed animals offers several advantages, including preservation of an intact circuitry and neurochemical milieu, and the ability to monitor activity at a scale that allows the early transition of local interictal spikes to propagating seizures to be detected. Hitherto, imaging of seizures in vivo has mainly relied on calcium-sensitive fluorescent reporters of neuronal activity (Rossi et al., 2017, 2018; Wenzel et al., 2017, 2019), which are sensitive but have limited temporal resolution, and have not been exploited extensively to distinguish between principal neurons and interneuron subtypes. Here we take an alternative approach to monitoring the transition from interictal to seizure activity, by imaging extracellular glutamate and GABA transients in the vicinity of a focus elicited by intracortical chemoconvulsant injection (Marvin et al., 2019). We show striking differences in the spatiotemporal evolution of the two neurotransmitters, and provide evidence that seizures are accompanied by a shift in the relative balance of glutamate and GABA transients. The data argue that the collapse of inhibitory restraint is at least partly due to a failure of GABA release.

\section{Results}

Extracellular GABA and glutamate imaging in acute chemoconvulsant epilepsy models

We imaged extracellular glutamate and GABA using variants of fluorescent reporters based on bacterial periplasmic binding proteins (Marvin et al., 2013, 2019) expressed in the visual cortex of adult mice by AAV injection. The glutamate sensors used here are collectively denoted iGluSnFR, and GABA sensors iGABASnFR, although several variants were tested, including $A A V$ vectors containing FLEXed constructs to allow Cre-dependent expression. Both sensors contained a PDGFR transmembrane domain, implying that they mainly sampled extrasynaptic neurotransmitter kinetics. Micron-scale imaging was not attempted, and instead we used spiral line scans across most of the field of view (512 x $512 \mu \mathrm{m})$ to characterize the time course of the average extracellular concentrations of GABA and glutamate approximately $100 \mu \mathrm{m}$ below the pia surface (Figure 1A-E). On the day of the experiment, the $\mathrm{GABA}_{A}$ receptor antagonist picrotoxin (PTX), or the cholinergic agonist 
pilocarpine, was injected into cortical layer 5 (at least $1 \mathrm{~mm}$ caudal to the imaging window, Figure 1A, C) to elicit seizures. By recording the electrocorticogram (ECoG) via an electrode implanted in the vicinity of the injection site, we obtained concurrent electrophysiological data on the evolution of epileptiform activity, which typically transitioned over $10-20$ minutes from IIDs to brief seizures lasting a few seconds, each of which was usually heralded by a sentinel spike which was indistinguishable from the IIDs (Figure 1F).

Temporal profiles of glutamate and GABA during IIDs

During the initial interictal spiking period, IIDs were accompanied by large amplitude iGluSnFR and iGABASnFR fluorescence transients (Figure 2A, B). The rise-time of iGABASnFR fluorescence transients (measured from 10\% to peak) was over two-fold slower than that of iGluSnFR transients (iGABASnFR: $134 \pm 10 \mathrm{~ms}$, average $\pm \mathrm{SEM}, \mathrm{n}=11$ mice; iGluSnFR: $63 \pm 6 \mathrm{~ms}, \mathrm{n}=14$ mice, $\mathrm{p}=2.15 \times 10^{-6}$, unpaired t-test), consistent with the slower binding and activation kinetics of iGABASnFR than iGluSnFR (Marvin et al., 2019). GABA sensor fluorescence transients also decayed more slowly than glutamate sensor transients (monoexponential $\tau$ fitted from peak to $1 \mathrm{~s}$ after the peak; iGluSnFR: $97 \pm 10 \mathrm{~ms}, \mathrm{n}=14$ mice; iGABASnFR: $288 \pm 28 \mathrm{~ms}, n=11$ mice; $p=1.2 \times 10^{-6}$ unpaired t-test, Figure $\left.2 \mathrm{C}, \mathbf{D}\right)$. As an alternative measure of kinetics, we integrated the decaying phase of fluorescence waveforms (area under the curve, AUC) and divided by the peak amplitude. This measure also indicated that the fluorescence kinetics of iGABASnFR were slower than those of iGluSnFR (iGluSnFR: $704 \pm 95$ ms vs iGABASnFR: $1450 \pm 37$ ms, $p=10^{-6}$ unpaired t-test, Figure $2 \mathrm{C}, \mathrm{E})$. This is unlikely to be due to an intrinsic difference in sensor kinetics because the decay time-constants previously estimated in vitro in response to electrical stimulation of neuronal cultures were similar for both sensors (Marvin et al., 2013, 2019), and similar to the decay for iGluSnFR during interictal spiking ( $\tau<100 \mathrm{~ms}$ ). The slower decay of iGABASnFR transients in vivo implies that GABA released during IIDs is cleared from the extracellular space more slowly than glutamate (It does not necessarily indicate that more GABA than glutamate is released because GABA transporters are expressed at a lower density than glutamate transporters (Chiu et al., 2002; Lehre and Danbolt, 1998; Scimemi, 2014)). 

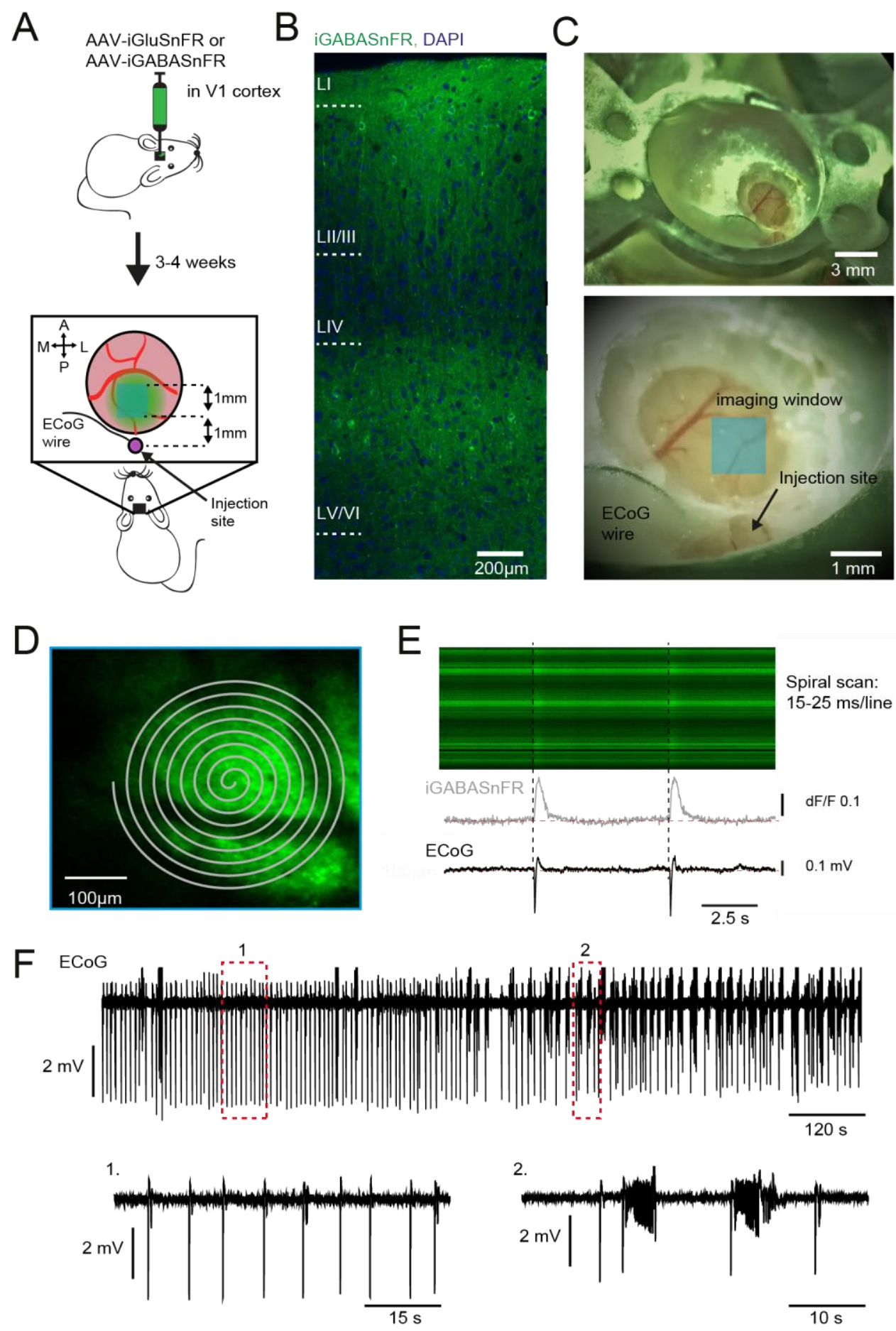

Figure 1: Protocol to characterize spatiotemporal neurotransmitter profiles during epileptic activity. (A) Experimental design. (B) Expression of iGABASnFR (green) and DAPI staining (blue) across layers of the visual cortex. The imaging plane was $\sim 100 \mu \mathrm{m}$ below the pia. (C) Photograph of the headpiece and craniotomy. The site of chemoconvulsant injection was $\sim 1 \mathrm{~mm}$ from the imaging window (blue square) and the ECoG electrode was positioned nearby. (D) iGABASnFR fluorescence from frame scan, with the spiral line scan trajectory superimposed. (E) Aligned linescan (top), average iGABASnFr signal and ECoG trace during interictal spiking. (F) Example of ECoG recording of epileptiform activity, initially dominated by IIDs (red rectangle 1 expanded below), which gave way to intermittent seizures lasting several seconds and heralded by sentinel spikes (red rectangle 2). 
The early kinetics of extracellular neurotransmitter concentrations following synaptic release are normally dominated by diffusion and binding to transporters, rather than by the much slower transport cycle (Diamond and Jahr, 1997). iGluSnFR itself can further buffer glutamate transients (Armbruster et al., 2020), and by analogy this may also occur for iGABASnFR and GABA. However, if GABA or glutamate release during IIDs overwhelms transporters, free neurotransmitter molecules may remain available for repeated binding to the fluorescent reporters. This leads to the prediction that, for any given experiment, the decay time of the iGluSnFR or iGABASnFR fluorescence transient should scale with the amount of neurotransmitter released. We therefore looked for a systematic relation between the decay time and the peak amplitude of individual IID-associated iGluSnFR or iGABASnFR fluorescence transients. Each value was normalized by the average of all events detected within each experiment to allow the data from different animals to be aggregated. We estimated the decay time of transients from AUC/peak amplitude because individual transients were sometimes too noisy to reliably fit a monoexponential curve and estimate $\tau$ directly. In keeping with the prediction, the decay times of both iGluSnFR and iGABASnFR transients were positively correlated with their peak amplitudes (Figure 2F). The relationship was steeper for iGABASnFR than for iGluSnFR transients, further arguing that GABA is cleared from the extracellular space more slowly than glutamate.

The data summarized above were obtained in experiments where either iGluSnFR or iGABASnFR was expressed and imaged separately. The availability of spectrally shifted variants of iGluSnFR (Marvin et al., 2018) permits, in principle, both neurotransmitters to be imaged simultaneously. We estimated the unmixing coefficients required to isolate the fluorescence of SF-Venus-iGluSnFR.A184V and GFP-iGABASnFR whilst imaging with three different emission filters (see Materials and Methods and Figure 2_S1). Simultaneous imaging of both sensors confirmed a slower decay of GABA than glutamate fluorescence in all 3 animals imaged (Figure 2_S1).

Taken together, these observations imply that IIDs are accompanied by massive GABA release and that GABA persists in the extracellular space for longer than glutamate. 
A

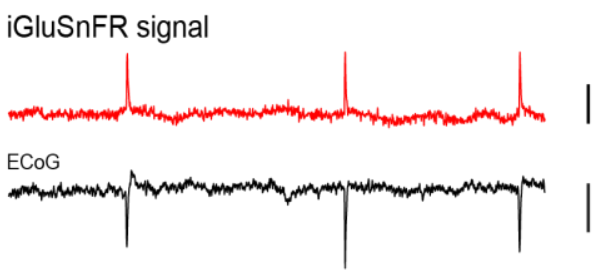

B

iGABASnFR signal

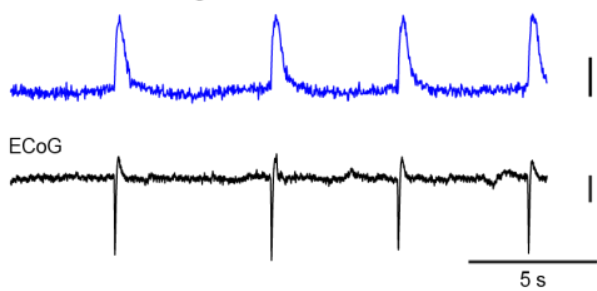

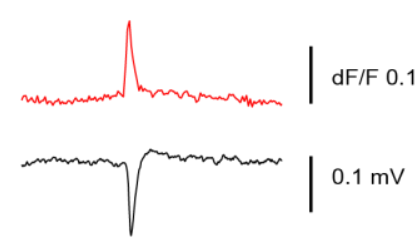
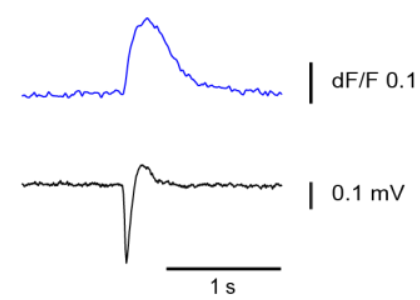

C
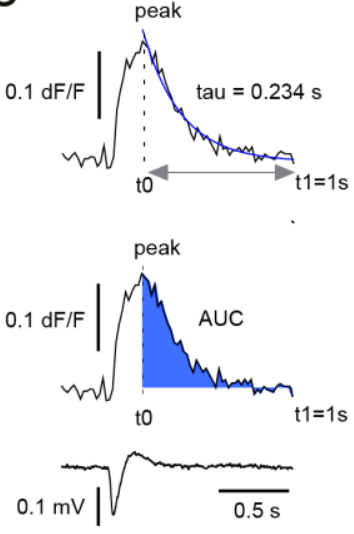

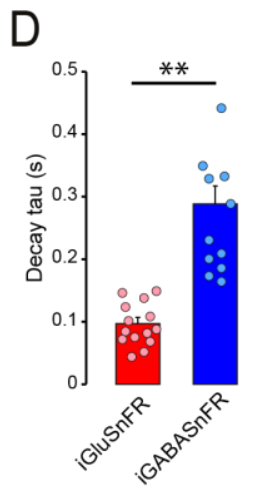

E

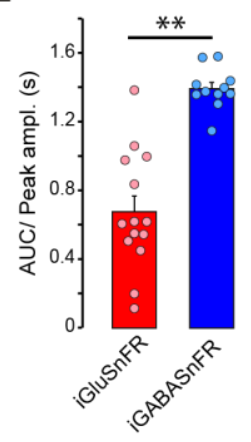

F iGluSnFR signal

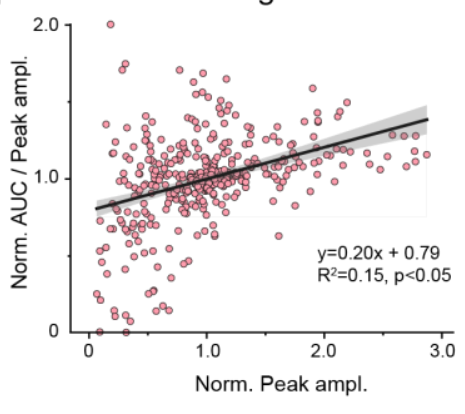

iGABASnFR signal

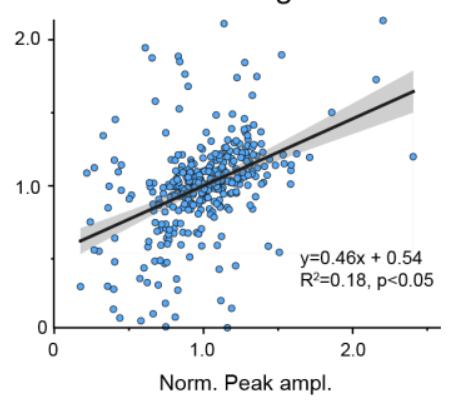

Figure 2: Extracellular GABA persists for longer than glutamate during IIDs. (A) iGluSnFR fluorescence and simultaneous ECoG. (B) Same for iGABASnFR. (C) Measurements of decay tau and the area under the curve from peak to 1s later (example trace from one iGABASnFR experiment). (D) Average decay tau of the glutamate and GABA transients for 14 mice expressing iGluSnFR and 11 expressing iGABASnFR. (E) Average area under the curve divided by peak amplitude (AUC/Peak ampl.) in the same experiments. (F) iGluSnFR (left panel) and iGABASnFR (right panel) fluorescence transient duration, expressed as AUC/Peak amplitude, plotted against peak amplitude, both normalized by the mean in each of 14 mice for iGluSnFR and 11 mice for iGABASnFR ( $n=391$ and 367 events, respectively). The lines show linear regressions with 95\% confidence intervals. Errors bars in (D) and (E) correspond to s.e.m.. ${ }^{* *} p<0.01$.

Spatial profiles of glutamate and GABA during IIDs

Previous $\mathrm{Ca}^{2+}$ imaging experiments using either pilocarpine or picrotoxin injection to evoke epileptiform activity showed that interictal events self-terminate and remain confined to a focus of radius $\sim 1 \mathrm{~mm}$, whilst other, initially indistinguishable, electrographic events turn into seizures that invade adjacent cortical territories (Rossi et al., 2017). We asked how neurotransmitter transients during IIDs relate to the distance from the ictal focus in the critical transition zone spanning $1 \mathrm{~mm}-2 \mathrm{~mm}$ from the site of chemoconvulsant application. Spiral scans were used to image iGluSnFR or iGABASnFR repeatedly at different locations 
within this interval (Figure 3_S1). In all but one experiment the peak intensity of the iGluSnFR fluorescence decreased monotonically with increasing distance from the injection site. In striking contrast, the peak of iGABASnFR fluorescence intensity was maximal at intermediate distances from the focus (Figure 3A-C). Expressed as a distance-weighted average within the imaging window, the GABA fluorescence transient was significantly further from the focus than the glutamate signal (iGluSnFR: $1.36 \pm 0.02 \mathrm{~mm}, \mathrm{n}=7$ mice; iGABASnFR: $1.46 \pm 0.02 \mathrm{~mm}, \mathrm{n}=5$ mice, $p=0.027$, unpaired t-test, Figure 3D). This held whether pilocarpine or picrotoxin was used to elicit IIDs (Figure 3, Figure 3_S1), and suggests that GABA release acts as an inhibitory halo around a hyperexcitable focus, analogous with feed-forward inhibition ahead of ictal wavefronts in epileptic patients and animal models (Liou et al., 2018; Schevon et al., 2012).

We estimated the direction of propagation of the waves of glutamate and GABA by calculating the time taken to reach $50 \%$ of the maximal fluorescence intensity at different regions in the imaging plane, and relating their locations to a vector that was rotated through $360^{\circ}$. The vector angle that yielded the steepest correlation between time-lag and distance along the vector defined the direction of propagation of the neurotransmitter wave (Figure S4_S1). This procedure also yielded an estimate of the speed of propagation of the interictal fluorescence transients. For iGluSnFR fluorescence transients, the wave propagated away from the focus, with a velocity of $65.5 \pm 12.8 \mathrm{~mm} / \mathrm{s}$ ( $\mathrm{n}=8 \mathrm{mice}$ ). For iGABASnFR, in contrast, the wave propagated towards the focus, at a significantly slower velocity $(27.6 \pm 8.7 \mathrm{~mm} / \mathrm{s}$, $n=7$ mice; $p=0.017$, Mann-Whitney test, Figure 4). This striking difference further supports the view that GABA release acts to constrain the spatial extent of IIDs. 


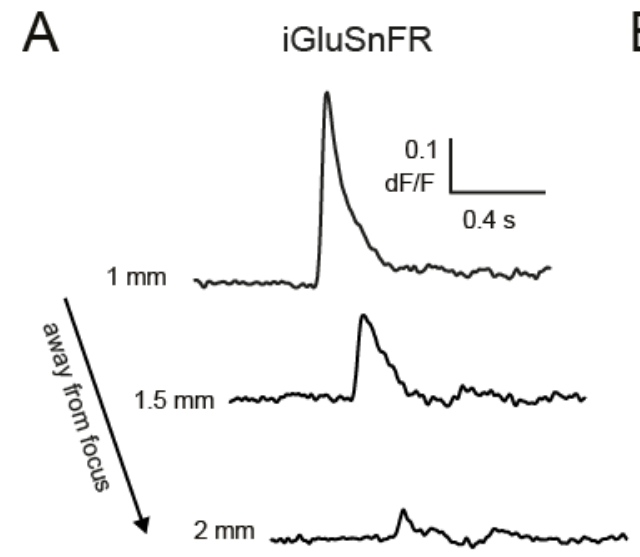

B iGABASnFR
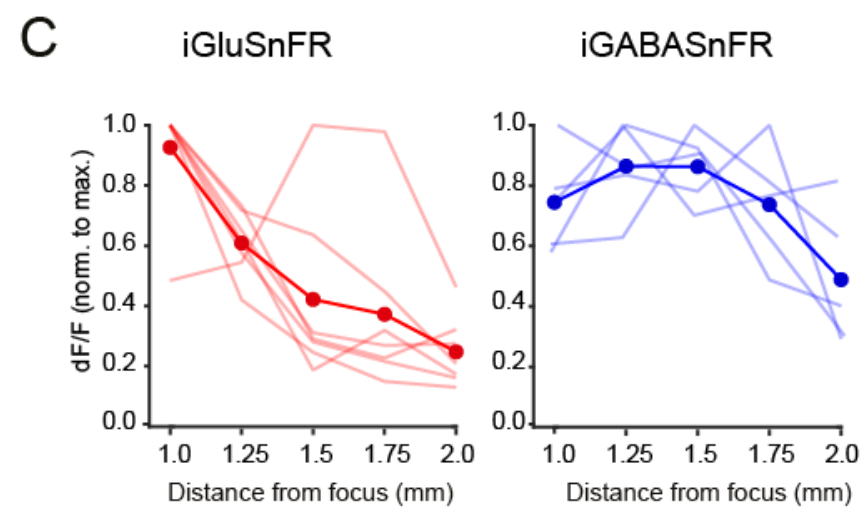

D

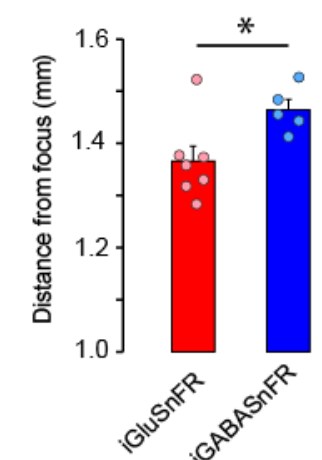

Figure 3: Contrasting spatial profile of glutamate and GABA during IIDs. (A)

Example iGluSnFR fluorescence transients at different distances from the focus during interictal spiking in one experiment. (B) Examples of iGABASnFR transients. (C) The iGluSnFR peak transient amplitude diminished with distance from the focus, whilst the iGABASnFR transient was maximal $\sim 1.5 \mathrm{~mm}$ away (light lines: individual experiments, thick line: average). In each experiment the spatial profile was normalized to the maximal fluorescence transient. (D) Peak-weighted average distance $\left(\sum\left(\left(\mathrm{dF} / \mathrm{F}^{*}\right.\right.\right.$ distance $\left.) / \Sigma(\mathrm{dF} / \mathrm{F})\right)$ of iGluSnFR and iGABASnFR transients within the imaging interval between $1 \mathrm{~mm}$ and $2 \mathrm{~mm}$ ( $\mathrm{n}=7$ mice for iGluSnFR and 5 mice for iGABASnFR). Errors bars in (D) correspond to s.e.m.. ${ }^{*} \mathrm{p}<0.05$. 
A

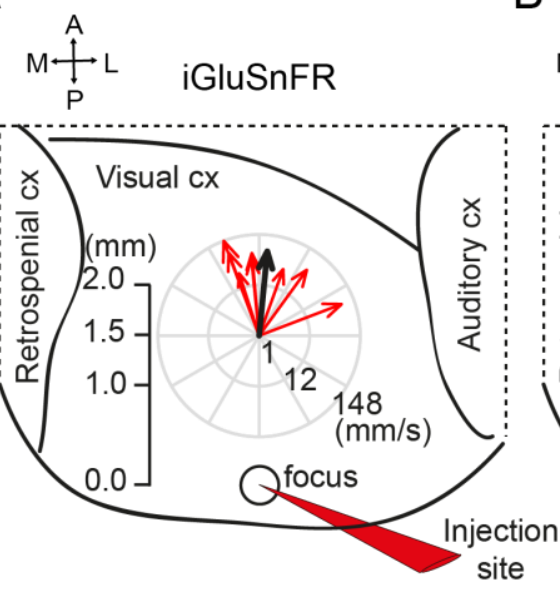

B
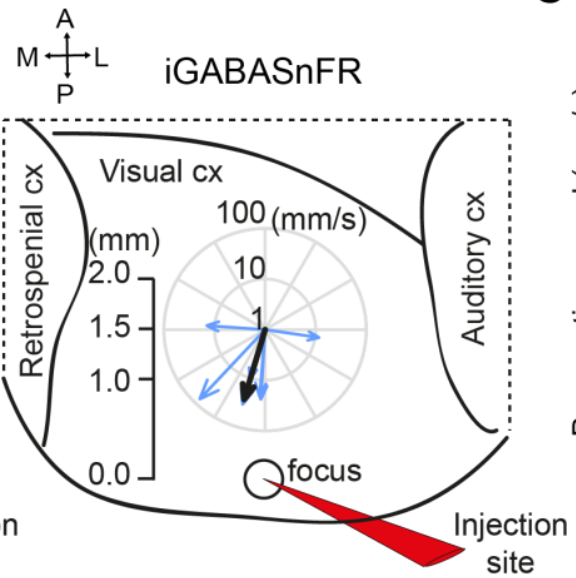

C

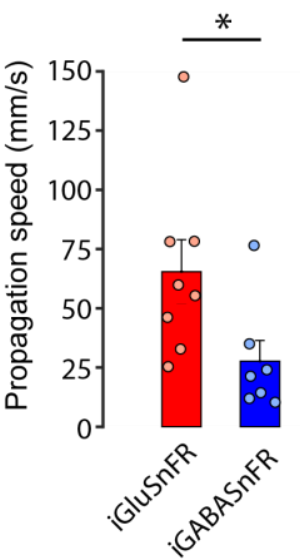

Figure 4: Glutamate and GABA waves oppose each other. (A) Propagation direction and velocity of the iGluSnFR signal in 8 animals (red arrows, average vector shown as black arrow) during IIDs, imaged $\sim 1.5 \mathrm{~mm}$ away from the focus. (B) Same but for iGABASnFR fluorescence transients. Glutamate and GABA waves travelled in opposite directions and at different velocities (note the logarithmic radial velocity scales in (A) and (B)). (C) Average propagation speed was significantly slower for the GABA waves. $n=8$ mice iGluSnFR (all with PTX) and $n=7$ mice for iGABASnFR ( 5 mice with PTX and 2 mice with pilocarpine). Errors bars in (C) correspond to s.e.m.. * $p<0.05$.

Interictal discharges gradually give way to seizures

The chemoconvulsant model studied here is characterized by a gradual increase in the frequency and duration of seizures, and a decrease in the occurrence of IIDs, over the first 30 minutes (Figure 5A). Previous in vitro studies have prompted the view that a progressive loss of preictal inhibitory barrages occurs over hundreds of seconds, facilitating the propagation of electrographic seizures (Trevelyan et al., 2006, 2007). We asked two questions. First, does iGABASnFR fluorescence shed light on why some events terminate immediately (IIDs) whilst others turn into seizures? And second, how do the spatial profiles of GABA and glutamate transients accompanying epileptiform events evolve on a timescale of minutes? For the latter purpose, we divided the period during which seizures occur into early ( $<500$ s from first electrographic seizure) and late ( $>500$ s from first seizure) epochs. The whole experiment was thus divided into initial interictal spiking (IIS), and early and late seizure activity (ESA and LSA, respectively) (Figure 5B-E). 
A

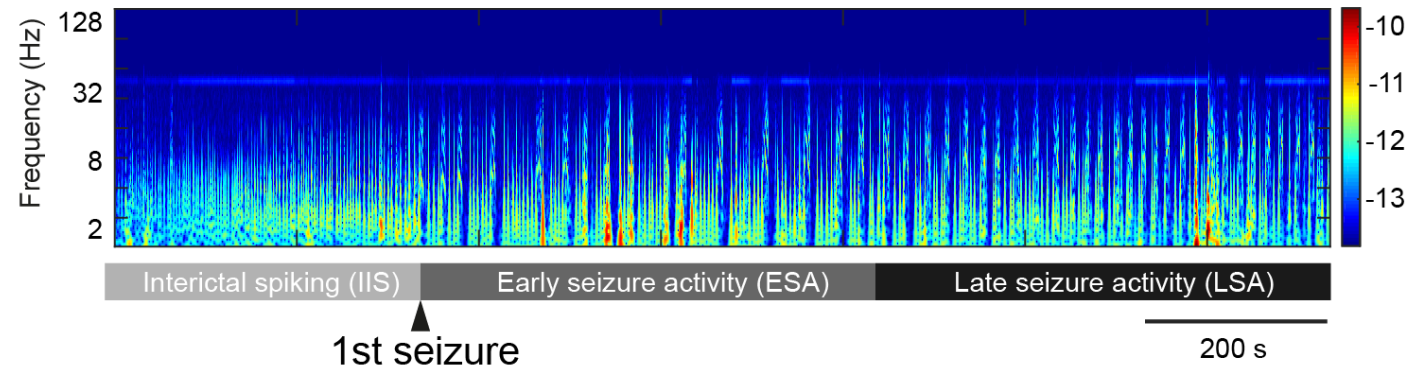

B

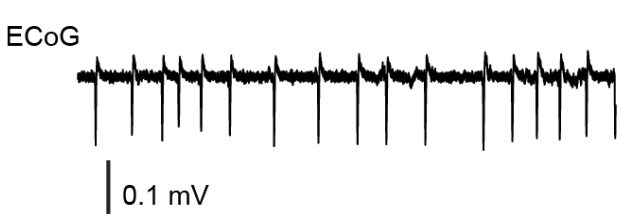

$C_{E C o G}$

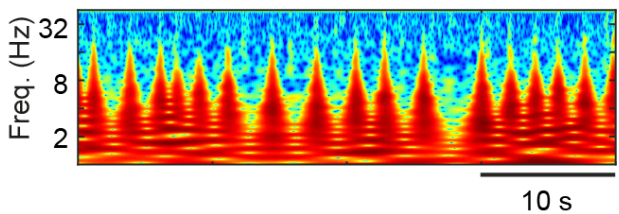

$\mathrm{D}$
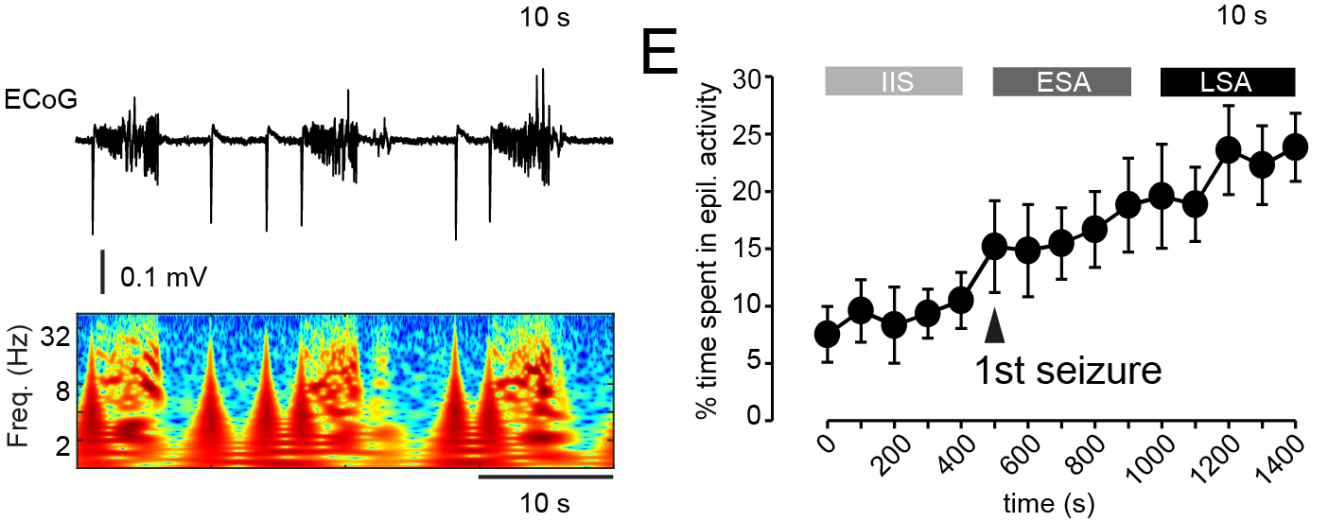

Figure 5: Evolution of epileptiform activity. (A) ECoG wavelet spectrogram divided in three periods: interictal spiking activity (IIS), and early and late seizure activity (ESA and LSA). (B) Representative ECoG and spectrogram during IIS. (C) and (D) Same as (B) but for ESA (C) and LSA (D). (E) Percentage of time spent in epileptiform activity (IIDs or seizures) over the three periods ( $n=8$ mice). Note the gradual increase with an abrupt transition after the first seizure.

Partial collapse of GABA release at seizure onset

What distinguishes IIDs from sentinel spikes that evolve into an ictus during ESA and LSA? We focused on iGABASnFR fluorescence transients imaged $\sim 1.5 \mathrm{~mm}$ from the focus, where maximal GABA response were detected during IIDs (Figure 3C, D). This coincides with the shortest distance at which cortical territory invasion can be detected with $\mathrm{Ca}^{2+}$ imaging, 
distinguishing seizures from IIDs (Rossi et al., 2017). We estimated the initial GABA release from iGABASnFR transients integrated for 300ms from their onset, before any additional spikes occurred in the ECoG (Figure 6A). GABA fluorescence transients thus measured were smaller when accompanying sentinel spikes than when associated with IIDs (sentinel spike to IID ratio: $0.860 \pm 0.048, p=0.024$, paired t-test, $n=8$ mice; Figure $6 B$ ). The peak amplitude of iGABASnFR transients was also significantly reduced at seizure onset (ratio: $0.866 \pm 0.045, p=0.021$, paired t-test, $n=8$ mice; Figure $6 C$ )

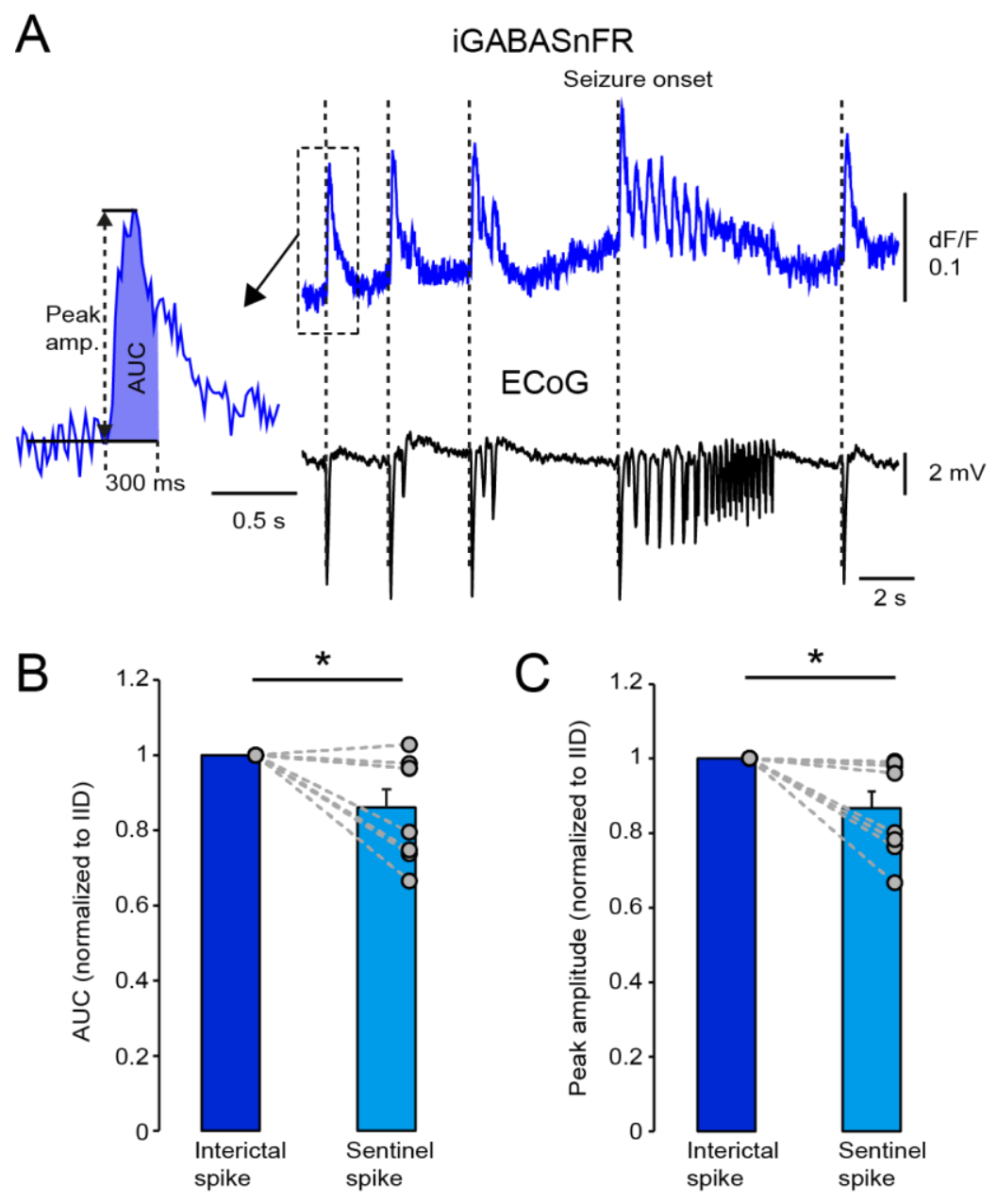

Figure 6: Decrease of iGABASnFR transient duration at seizure onset. (A) Single example of iGABASnFR fluorescence and ECoG trace. The left panel indicates the peak amplitude of the GABA transients as well as the AUC measured from onset to 300 ms later. (B) Average of AUC and $(\mathbf{C})$ peak amplitude during IIDs and the sentinel spike heralding a seizure. $n=8$ mice, Errors bars in (B) and (C) correspond to s.e.m.. ${ }^{*} \mathrm{p}<0.05$.

On occasion, we were able to follow the evolution of GABA transients for several consecutive IIDs before and after a seizure. We restricted attention to sequences consisting of 3 interictal spikes before, and one after, a sentinel spike and seizure. This revealed a 
decrease of GABA release before seizure onset, with recovery after the seizure ( 3 sequences per mice, 3 mice, Supp Figure S6_1).

Taken together, these findings imply that seizure onset is accompanied by a partial failure of GABA release, providing a parsimonious explanation for an escape of epileptiform activity from surround inhibition.

Slow erosion of GABA release accompanies increased invasion of the neighboring cortical territory

At any stage of the gradual evolution through ESA and LSA (Figure 5), the spatial profiles of GABA or glutamate transients accompanying sentinel spikes were similar to those corresponding to intercurrent IIDs. We therefore averaged them together in order to study the gradual evolution of the amplitude and shape of glutamate and GABA profiles (Figure 7_S1). iGluSnFR transients increased significantly as the cortical activity gradually evolved from IIS through ESA to LSA (AUC: $F[1,5]=249.9, p=1.8 \times 10^{-5}, n=6$ mice, RM-ANOVA, Figure 7A). The increase in glutamate transients was most marked as IIS gave way to ESA (AUC ESA/IIS: $2.79 \pm 0.21, p=10^{-4}$; LSA/IIS: $3.76 \pm 0.30, p=2.2 \times 10^{-6}, n=6$ mice, Bonferroni test, Figure 7A). In addition, the inverse relationship between amplitude and distance seen for iGluSnFR fluorescence during IIS (Figure 3) disappeared (Figure 7B). The greater invasion of the cortex implies a collapse of the spatial constraint of glutamatergic signaling. Interestingly, this pattern was observed whether restricting attention to the sentinel spikes of individual brief seizures or to the interictal spikes that continued to occur in between seizures (Figure 7_S1).

In contrast to glutamate transients, the iGABASnFR signal diminished as IIS gave way to ESA and LSA (AUC: $F[1,6]=224.2, p=5.5 \times 10^{-6}, n=7$ mice, RM-ANOVA, AUC ESA/IIS: $0.81 \pm$ $0.08, p=0.15$; LSA/IIS: $0.70 \pm 0.10, p=0.01, n=7$ mice, Bonferroni test, Figure $7 \mathrm{C})$. The relationship between peak amplitude and distance showed a tendency to flatten out (Figure 7D).

Taken together, these results confirm a progressive erosion of inhibition during seizure activity close to the focus, and show that this is mediated at least in part by a decrease in GABA release in an annulus surrounding the focus. Conversely, glutamate imaging shows 
that seizures are associated with invasion of new territories as expected from the recruitment of excitatory neurons.

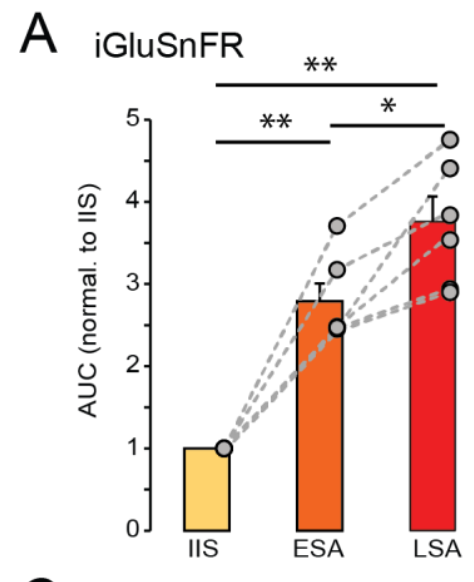

B iGluSnFR
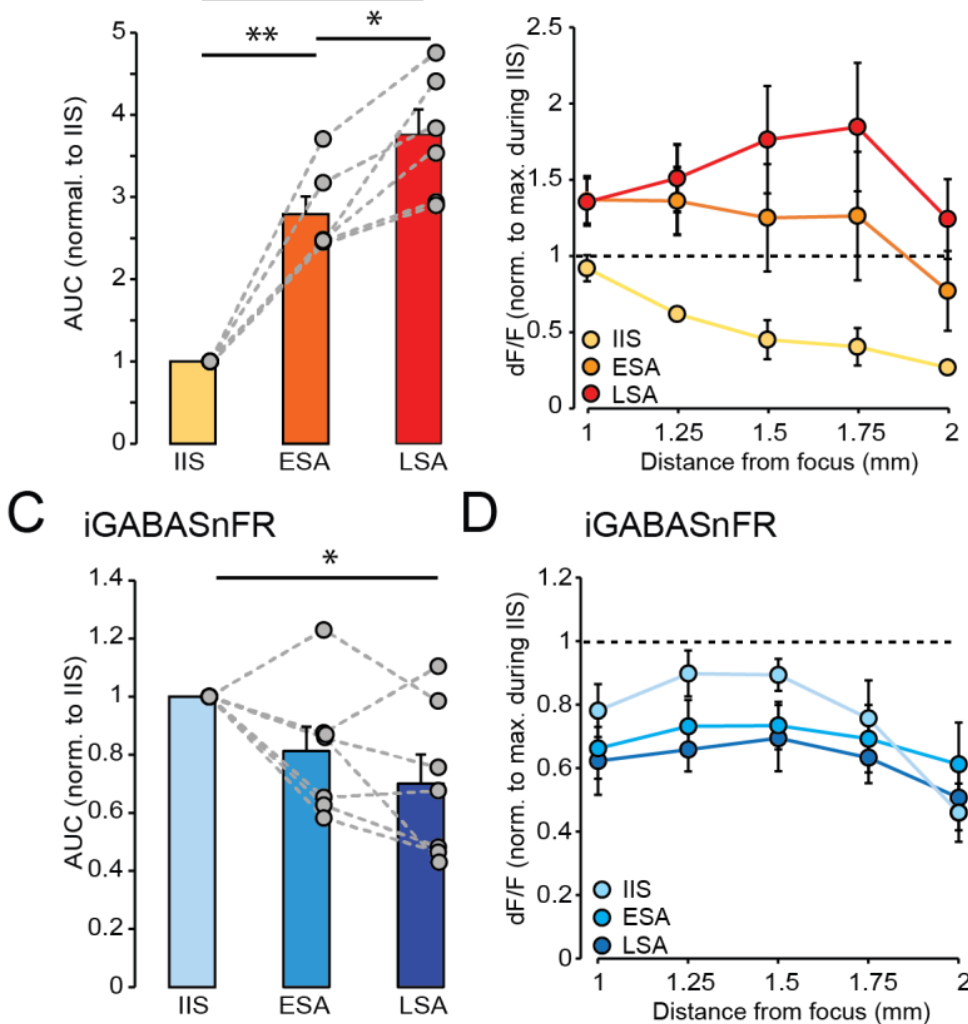

D IGABASnFR

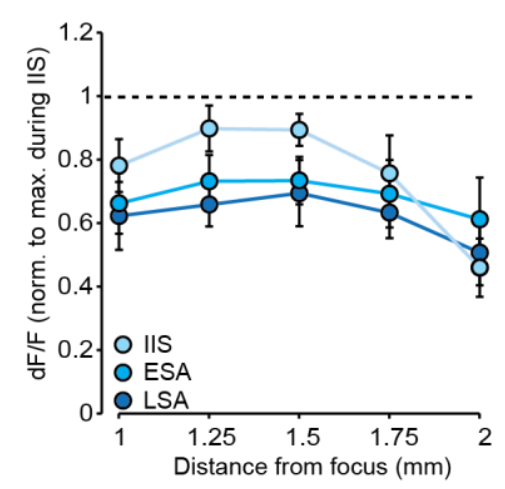

Figure 7: Rapid increase of glutamate release and progressive decrease of GABA release during evolution from spiking to seizures. (A) AUC of iGluSnFR transients during IIS, ESA and LSA. Note the rapid increase of glutamate release during the early phase of seizure activity ( $n=6$ mice). (B) iGluSnFR transients increased in amplitude and broadened out as seizures took over from spikes ( $n=6$ mice). (C) Same as $A$ but for iGABASnFR. Note the gradual decrease of GABA release ( $n=7$ mice). (D) The spatial profile of iGABASnFR transients showed little change $(n=5$ mice during IIS and ESA, and 4 mice during LSA). Errors bars correspond to s.e.m.. ${ }^{* *} p<0.01,{ }^{*} p<0.05$.

\section{Discussion}

The present study reveals strikingly different profiles of extracellular GABA and glutamate during interictal discharges. Whilst glutamate pulses propagate centrifugally, GABA transients are maximal in an annulus surrounding the focus, consistent with recruitment of feed-forward interneurons confining excessive activity and preventing runaway excitation. IIDs are superficially indistinguishable from sentinel spikes of seizures. However, GABA- 
dependent fluorescence transients at the onset of seizures are smaller, providing an explanation why epileptiform activity fails to be contained by feed-forward inhibition. As interictal spiking gradually gives way to seizures, GABA transients further diminish, consistent with a progressive erosion of inhibitory restraint.

Although the concept of an inhibitory restraint is not novel, the experimental evidence to date comes from electrophysiological recordings and $\mathrm{Ca}^{2+}$ imaging, both of which have limitations. Electrophysiology samples the cortex sparsely, although recently developed high-density probes are beginning to shed light on the activity of multiple neurons in a small area (Jun et al., 2017). Identifying action potentials generated by individual neurons through the evolution of a seizure is however complicated by poor signal to noise ratios and distortion of the extracellular fields, and the full potential of such high-density probes remains to be determined. $\mathrm{Ca}^{2+}$ imaging, on the other hand, generally has a slow temporal resolution dictated by the off-rate of fluorescent indicators (Kerruth et al., 2019), and has not yet been multiplexed to allow simultaneous imaging of different neuronal subtypes. Recently optimized voltage indicators show promise because of their fast kinetics and improved dynamic range (Abdelfattah et al., 2019; Piatkevich et al., 2019), but have yet to be applied to understand seizure mechanisms. Importantly, intracellular $\mathrm{Ca}^{2+}$ elevation and neuronal depolarization can occur in the absence of neurotransmitter release if neurons enter a state of depolarization block. Fluorescence imaging using iGluSnFR and iGABASnFR fills an important gap in that it provides a direct measure of the interplay of excitatory and inhibitory neurotransmitter release.

Previous single cell electrophysiology studies have shown that pyramidal neurons receive predominantly synchronous GABAergic currents during IIDs elicited by chemoconvulsants and/or pro-convulsant perfusion solutions. This has been reported both in brain slices obtained from rodents (Karlócai et al., 2014; Trevelyan et al., 2006, 2007; Zhang et al., 2012) and in tissue resected from epileptic patients (Cohen, 2002; Huberfeld et al., 2011). Synchronous $\mathrm{Ca}^{2+}$ transients in interneurons also occur during interictal spikes in a chronic epilepsy model (Muldoon et al., 2015). Our imaging data extend these findings by revealing intense GABA release during IIDs, with iGABASnFR fluorescence timecourse suggestive of slow neurotransmitter clearance, but also show quasi-synchronous glutamate release. A striking difference, however, is that the glutamate transients diminished in peak amplitude with increasing distance from the focus, whilst GABA transients were maximal $1.5 \mathrm{~mm}$ from the site of injection. In addition, the iGluSnFR and iGABASnFR fluorescence waves associated with IIDs propagated at different velocities and in opposite directions: rapidly and centrifugally in the case of glutamate and more slowly and centripetally in the case of GABA. 
A caveat should be stressed, however: we were unable to image the neurotransmitters at the core of the ictal focus. Nevertheless, the iGluSnFR and iGABASnFR fluorescence recordings provide direct evidence for surround inhibition (Prince and Wilder, 1967) constraining epileptiform activity inside the focus. It also confirms that a similar inhibitory restraint phenomenon takes place during IIDs at the focus as observed during ictal propagation in animal models and epileptic patients (Liou et al., 2018; Schevon et al., 2012). Notably, the glutamate waves observed in our study propagated at a similar speed ( 100 $\mathrm{mm} / \mathrm{s}$ ) as the LFP in some patients with epilepsy in whom grids of electrodes were used to capture the propagation of focal cortical seizures (Schevon et al., 2012). The GABA wave was 2 to 3 times slower. A simple model to account for the results is that a 'detonation' of glutamatergic neurons at the focus recruits feedforward interneurons, resulting in an annulus of GABA release. Depolarization block of proximal interneurons (Karlócai et al., 2014) could then explain why less GABA is released closer to the focus.

A striking finding in the present study was that sentinel spikes heralding seizures onset were accompanied by smaller iGABASnFR transients than IIDs. A previous study using $\mathrm{Ca}^{2+}$ fluorescence imaging in pyramidal neurons in a similar awake head-fixed model failed to distinguish between IIDs and seizures during the first 300ms (Rossi et al., 2017). A partial failure of GABA release is a strong candidate explanation for why inhibitory restraint collapses intermittently. Another in vitro study using low $\left[\mathrm{Mg}^{2+}\right]$ to evoke epileptiform activity reported a gradual reduction of synchronous GABAergic currents in pyramidal cells and interneurons starting between 3 and 5 IIDs prior to seizure onset (Zhang et al., 2012). This remarkably matches our observations in three animals where we were able to capture a sufficient number of events (Figure 6_S1). Although the data are observational, they strongly suggest that a decrease in GABA release is causally upstream of the transition of IIDs to seizures. We also observed a gradual decrease of GABA release and a broadening of the glutamate waves with each event (whether IID or sentinel spike) accompanying the evolution of seizure activity over minutes. This pattern is in accord with a progressive loss of inhibitory barrages seen in acute slices while the frequency of ictal events increased (Trevelyan et al., 2007).

Why does GABA release partially collapse with the discharges leading up to seizures? Although depolarization block of interneurons (Cammarota et al., 2013; Karlócai et al., 2014) could account for this observation, it could, in principle, also be explained by vesicle depletion (Zhang et al., 2012) or presynaptic inhibition of GABAergic terminals (Lei and McBain, 2003; Overstreet-Wadiche and McBain, 2015). These phenomena are not mutually exclusive, and all three could, in principle, occur, especially when taking into account the 
diversity of interneurons. However, vesicle depletion and presynaptic autoinhibition mechanisms typically require repeated spiking activity above $10 \mathrm{~Hz}$ (Fernández-Alfonso and Ryan, 2004; Lei and McBain, 2003; Yamashita et al., 2018), and such short-term depression would be expected to have faded before the next interictal spike. We tentatively suggest that intense depolarization, preventing a fraction of interneurons from firing, is the most plausible explanation for the partial collapse of GABA release at seizure onset. Although this mechanism has the attraction that it also explains why GABA release is maximal in an annulus around the ictal core, it remains to be tested directly.

Our experimental design only allowed us to image the very superficial cortical layers and therefore to study extracellular glutamate and GABA release on distal dendrites from pyramidal neurons. Future investigations to study the glutamate and GABA distributions during epileptic activity across cortical layers would be of great interest (Wenzel et al., 2017). In addition, identifying the sources of the GABA transients would shed light on whether the partial collapse of GABA release is specific to a particular interneuron subtype. Layer 1 includes at least 4 different types of interneurons (Schuman et al., 2019) as well as axonal terminals from somatostatin-positive interneurons (Tremblay et al., 2016), and so there are several candidates. A further caveat is that the present study used acute chemoconvulsant models. However, the ability to model focal cortical dysplasia, which is associated with spontaneous seizures (Hsieh et al., 2016), as well as open-source miniaturized microscopes (Cai et al., 2016; Shuman et al., 2020), may allow glutamate and GABA release to be studied in chronic epilepsy models.

To conclude, we have provided direct evidence for collapse of an inhibitory restraint in awake animals. Future investigation of the precise mechanisms underlying this failure, and the interneurons responsible, may help to design new strategies to prevent epileptiform activity from invading new territories. 


\section{Methods}

\section{Animals}

All experimental procedures on animals were conducted according to the UK Animals Scientific Procedures Act (1986). Experiments were performed at University College London, under personal and project licenses released by the Home Office following appropriate ethics review. CaMKII-Cre (B6.Cg-Tg(Camk2a-cre)T29-1Stl/J, stock no: 005359, Jackson laboratory) or wild-type C57BL/6 (Harlan) mice were transfected locally with an adeno-associated viral vector carrying either iGluSnFR or iGABASnFR constructs. Animals were housed on $12 \mathrm{~h} / 12 \mathrm{~h}$ dark/light cycle, and food and water were given ad libitum.

\section{Surgery and viral injection}

Animals of both sexes (P30-50) were deeply anaesthetized in an induction chamber with 4$5 \%$ isoflurane. They were then transferred into a stereotaxic frame (David Kopf Instruments Ltd., USA) and maintained under anaesthesia with 1.5-2.5\% isoflurane. Buprenorphine (0.02 $\mathrm{mg} / \mathrm{kg})$ and metacam $(0.1 \mathrm{mg} / \mathrm{kg})$ were injected subcutaneously before starting any surgery while dexamethasone $(2 \mathrm{mg} / \mathrm{kg})$ was administered by intramuscular injection before performing the craniotomy. In order to image iGABASnFR or iGluSnFR, animals were subjected to two procedures.

First, mice were injected in layer 5 of the visual cortex using a microinjection pump (WPI Ltd., USA), $5 \mu$ l Hamilton syringe (Esslab Ltd., UK), and a 33 gauge needle (Esslab Ltd., UK) (injection volume per site: $350 \mathrm{~nL}$, rate: $100 \mathrm{~nL} / \mathrm{min}$ ) with either AAV2/5.hSyn.Flex.iGluSnFr.WPRE.SV40 (titre: $2.5 \times 10^{13} \mathrm{GC}$ per ml, Penn Vector Core) or AAV2/1.SynFLEX.iGABASnFR.F102G.mRuby3 (titre: $1.0 \times 10^{13} \mathrm{GC}$ per $\mathrm{ml}$, Janelia Farm). In some cases, animals were injected with either an AAV2/1.SynFLEX.iGABASnFR.F102G (titre: $1.0 \times 10^{13} \mathrm{GC}$ per ml, Janelia Farm) or a mixture (1:1) of AAV2/1.hSyn-SF-VenusiGluSnFR.A184V (titre: $9.5 \times 10^{12} \mathrm{GC}$ per ml Janelia Farm) and AAV2/1.SynFLEX.iGABASnFR.F102G.mRuby3 (titre: 1.0x10 13 GC per ml, Janelia Farm). After $10 \mathrm{~min}$, the needle was retracted slowly to the surface, the animal sutured and given 0.5-1 mL sterile saline subcutaneously. Mice were checked daily for several days postsurgery. 
Three to four weeks post-injection, animals were chronically implanted with a head-post (Model 9, Neurotar Ltd) and cranial window of $2 \mathrm{~mm}$ in the vicinity of the viral injection site was performed using a punch hole (Kain medical). The craniotomy was then covered with a coverslip (3mm) and glued in place. Posterior to the cranial window a small burr hole was drilled for future injection of chemoconvulsant drugs and stainless-steel wire $(0.075 \mathrm{~mm}$, Advent Ltd) was implanted at the cortical surface in the close proximity to record the ECoG (Figure 1). A reference electrode was implanted in the contralateral frontal lobe. The headpost, electrodes and connectors were secured by opaque dental cement, and silicone elastomer sealant (Kwik-Cast, World Precision Instruments) was used to cover and seal the entire chamber area in order to protect the imaging window and site of injection (Figure 1). Animals were then allowed to recover before being habituated in the head-fixed system (Neurotar Ltd).

\section{Simultaneous imaging and ECoG recordings}

Imaging data were acquired on an FV1200MPE multi-photon laser scanning microscope with an in vivo X25 objective (XLPLN25XWMP2, Olympus), coupled to a Ti-sapphire Chameleon Ultra pulsed laser (Coherent), and adapted to allow a head-fixed system (Neurotar Ltd). iGABASnFR and iGluSnFR were excited at $920 \mathrm{~nm}$ and emission fluorescence was collected via 3 PMTs and filters (PMT 1: 450-500 nm; PMT 2: 515-560 nm; PMT 3: 590-650 nm). iGluSnFR and iGABASnFR imaging was performed by using a spiral line scan at $40-60 \mathrm{~Hz}$ at $320 \times 320$ pixel $(512 \times 512 \mu \mathrm{m}$, Figure 1) and laser power was kept constant within each experiment. Each imaging acquisition lasted $\sim 20 \mathrm{sec}$ during the initial phase and was increased to $\sim 40 \mathrm{sec}$ after detection of the first seizure. Three overlapping imaging loci between 1 and $2 \mathrm{~mm}$ from the injection site, covering approximatively $1 \mathrm{~mm}$ in the antero-posterior axis, were selected before starting each recording session (Figure 1,

Figure 3-S1). The center points of each imaging locus, and distances from the chemoconvulsant injection site, were recorded from the $X, Y$ coordinates given by a motorized platform (Scientifica) holding the head clamp (Neurotar Ltd). Different areas were repeatedly imaged in a pseudorandom order.

The ECoG signal was acquired during the entire experiment via a MultiClamp 700B amplifier (Molecular Devices) and time-stamped with the imaging data by continuously recording the TTL output signal from the scanning-head. ECoG data were bandpass-filtered from 0.1 to $300 \mathrm{~Hz}$, digitized at $1 \mathrm{kHz}$ and recorded using WinEDR open source software (Strathclyde University, UK). 


\section{Acute experiment protocol}

After three or four days of habituation to the head-restraint system, animals were lightly anesthetised ( $1.5 \%$ isoflurane) and placed in a stereotaxic frame (Kopf Instruments) for the chemoconvulsant injection. After removing the silicone membrane covering the chamber and inspection of the craniotomy, an Hamilton syringe needle (33 gauge) was inserted into the brain via the injection site hole to a depth of $500-600 \mu \mathrm{m}$ below the pia (corresponding to layer 5, Figure 1). Either picrotoxin (PTX, 10mM, 50-100nL) or pilocarpine (3.5M, 150$300 \mathrm{~nL}$ ) diluted in sterile saline was injected at $100 \mathrm{~nL} / \mathrm{min}$, and the needle was removed $\sim 100$ seconds later. The animal was then transferred to the imaging set-up to start the recording session. During the session, animals were checked intermittently for signs of distress and given water via a $0.5 \mathrm{~mL}$ syringe. Typically, a recording session lasted up to 30 40 min from the chemoconvulsant injection. If the animal was still seizing at the end of the session, it was given diazepam (10 mg/kg) before returning it to its home-cage. All animals were monitored for several hours after each imaging session. Most data were acquired during a single imaging session. However, in few animals where the chemoconvulsants failed to induce seizures, a second session was performed few days later.

\section{Analysis}

\section{Image analysis}

Imaging data were initially screened using ImageJ software, and the fluorescence timecourses from each pixel extracted for further analysis in MATLAB (The MathWorks Inc.). All analyses were performed using custom-code MATLAB or Python scripts. Briefly, the average fluorescent signal from each scan line was aligned to the ECoG traces by using the recorded TTL output pulse from head-scanning system and each fluorescent point was placed at the centre of TTL pulse in the electrophysiological recordings. For each trace, dF/F was calculated by taking the baseline $\mathrm{F}$ as the mean fluorescence during the entire imaging acquisition (either 20 s or 40 s, see above).

Average fluorescent signals were aligned to the onset of interictal spikes or sentinel spikes (for seizures) as determined from the electrophysiological trace. To estimate the decay time constant of the iGABASnFR and iGluSnFR signal during interictal spikes, a single monoexponential curve was fitted (Figure 2) from the peak to 1s after the peak. 
Measurement of the area under the curve (AUC) of the iGABASnFR signal associated with interictal spikes or sentinel spikes was restricted to the first $300 \mathrm{~ms}$ to avoid later inflexions of the ECoG (Figure 6). In this case, all fluorescent transients were collected at $\sim 1.5 \mathrm{~mm}$ from the focus.

To unmix the fluorescence signals arising from simultaneous imaging of iGABASnFR and iGluSnFR signals (Figure 2-S1), we first established, in separate experiments, the fluorescent profile of each probe alone collected by the three PMTs (Figure 2_S1 A). This allowed us to determine the proportion of fluorescent signal detected by each PMT during interictal spikes for iGABASnFR and a Venus version of iGluSnFR. Hence, we found that the relative Venus-iGluSnFR signals acquired via $\mathrm{PMT}_{1}, \mathrm{PMT}_{2}$ and $\mathrm{PMT}_{3}$ were $~ 0: 1: 0.25$, and the relative iGABASnFR signals were $\sim 1: 1: 0$. We then used these profiles to estimate the contribution of both probes expressed simultaneously, and noise, to the fluorescent signals collected via each PMT as follows: $F_{\text {PMT1 }}=\left(F_{\text {iGABASnFR }}+\right.$ noise $), F_{\text {PMT2 }}=\left(F_{\text {Venus-iGluSnFR }}+\right.$ $F_{\text {iGABASnFR }}+$ noise $)$ and $F_{\text {PMT3 }}=\left(0.25^{*} F_{\text {Venus-iGluSnFR }}+\right.$ noise $)$. From these formulas, we then separated the Venus-iGluSnFR signal from iGABASnFR fluorescence trace as follows: $F_{\text {Venus-iGluSnFR }}=\left(F_{\text {PMT2}^{-}} F_{\text {PMT1 } 1}\right)$ and $F_{\text {IGABASnFR }}=\left(A^{*}\left(F_{\text {PMT1 } 1}\right)+\left(F_{P M T 2}\right) / B-F_{\text {PMT3 }}\right) ;$ where $A=0.736$ and $\mathrm{B}=3.8$.

To determine the direction of propagation and velocity of fluorescent signals, we first realigned the fluorescence timecourse for each pixel to compensate for the inter-pixel interval $(10 \mu \mathrm{s})$. We then estimated the time at which regions opposite one another in the imaged area (equidistant from its center, $\sim 1.5 \mathrm{~mm}$ from the focus) reached $50 \%$ of maximal fluorescence intensity during each IIS $\left(T_{50}\right)$. A vector in the $X-Y$ plane passing through the opposite regions was rotated through $360^{\circ}$, and the vector angle that yielded the steepest correlation between $\mathrm{T}_{50}$ and the distance along the vector defined the direction of propagation of the neurotransmitter wave (Figure 4-S1). This procedure also allowed us to estimate the speed of propagation of the interictal fluorescence transients.

\section{Chemicals}

Picrotoxin and pilocarpine hydrochloride were purchased from Tocris (CAS number: 124-878) and Sigma-Aldrich (CAS number: 54-71-7) respectively and diluted on the day of the experiment in sterile saline.

\section{Statistics}

All statistical analyses were performed using either OriginPro software, or in MATLAB. Normal distribution was tested for each data set and parametric or non-parametric test were 
used appropriately. Paired and unpaired t-test were always two-tailed. For the angle and direction of the iGABASnFR and iGluSnFR signal propagation, angles were averaged for each animal and compared using Parametric Watson-Williams multi-sample test. $\mathrm{N}$ values used in statistical analysis represent the number of animals in each experimental group.

Data are shown as mean + -- s.e.m.. Statistical differences were considered significant at $p<$ 0.05 . 


\section{References}

Armbruster, M., Dulla, C.G., and Diamond, J.S. (2020). Effects of fluorescent glutamate indicators on neurotransmitter diffusion and uptake. ELife 9, e54441.

Avoli, M., Louvel, J., Kurcewicz, I., Pumain, R., and Barbarosie, M. (1996). Extracellular free potassium and calcium during synchronous activity induced by 4-aminopyridine in the juvenile rat hippocampus. The Journal of Physiology 493, 707-717.

Bohannon, A.S., and Hablitz, J.J. (2017). Optogenetic dissection of roles of specific cortical interneuron subtypes in GABAergic network synchronization. The Journal of Physiology 596, 901-919.

Cai, D.J., Aharoni, D., Shuman, T., Shobe, J., Biane, J., Song, W., Wei, B., Veshkini, M., LaVu, M., Lou, J., et al. (2016). A shared neural ensemble links distinct contextual memories encoded close in time. Nature 534, 115-118.

Cammarota, M., Losi, G., Chiavegato, A., Zonta, M., and Carmignoto, G. (2013). Fast spiking interneuron control of seizure propagation in a cortical slice model of focal epilepsy. J Physiol 591, 807-822.

Chiu, C.-S., Jensen, K., Sokolova, I., Wang, D., Li, M., Deshpande, P., Davidson, N., Mody, I., Quick, M.W., Quake, S.R., et al. (2002). Number, Density, and Surface/Cytoplasmic Distribution of GABA Transporters at Presynaptic Structures of Knock-In Mice Carrying GABA Transporter Subtype 1-Green Fluorescent Protein Fusions. J. Neurosci. 22, 1025110266.

Cohen, I. (2002). On the Origin of Interictal Activity in Human Temporal Lobe Epilepsy in Vitro. Science 298, 1418-1421.

de Curtis, M., and Avanzini, G. (2001). Interictal spikes in focal epileptogenesis. Progress in Neurobiology 63, 541-567.

Diamond, J.S., and Jahr, C.E. (1997). Transporters Buffer Synaptically Released Glutamate on a Submillisecond Time Scale. J. Neurosci. 17, 4672-4687.

Fernández-Alfonso, T., and Ryan, T.A. (2004). The Kinetics of Synaptic Vesicle Pool Depletion at CNS Synaptic Terminals. Neuron 41, 943-953.

Hsieh, L.S., Wen, J.H., Claycomb, K., Huang, Y., Harrsch, F.A., Naegele, J.R., Hyder, F., Buchanan, G.F., and Bordey, A. (2016). Convulsive seizures from experimental focal cortical dysplasia occur independently of cell misplacement. Nature Communications 7, 1-12.

Huberfeld, G., Menendez de la Prida, L., Pallud, J., Cohen, I., Le Van Quyen, M., Adam, C., Clemenceau, S., Baulac, M., and Miles, R. (2011). Glutamatergic pre-ictal discharges emerge at the transition to seizure in human epilepsy. Nature Neuroscience 14, 627-634.

Jun, J.J., Steinmetz, N.A., Siegle, J.H., Denman, D.J., Bauza, M., Barbarits, B., Lee, A.K., Anastassiou, C.A., Andrei, A., Aydın, Ç., et al. (2017). Fully integrated silicon probes for high-density recording of neural activity. Nature 551, 232-236.

Karlócai, M.R., Kohus, Z., Káli, S., Ulbert, I., Szabó, G., Máté, Z., Freund, T.F., and Gulyás, A.I. (2014). Physiological sharp wave-ripples and interictal events in vitro: what's the difference? Brain 137, 463-485. 
Kerruth, S., Coates, C., Dürst, C.D., Oertner, T.G., and Török, K. (2019). The kinetic mechanisms of fast-decay red-fluorescent genetically encoded calcium indicators. J. Biol. Chem. 294, 3934-3946.

Lehre, K.P., and Danbolt, N.C. (1998). The Number of Glutamate Transporter Subtype Molecules at Glutamatergic Synapses: Chemical and Stereological Quantification in Young Adult Rat Brain. J Neurosci 18, 8751-8757.

Lei, S., and McBain, C.J. (2003). GABAB receptor modulation of excitatory and inhibitory synaptic transmission onto rat CA3 hippocampal interneurons. J Physiol 546, 439-453.

Lévesque, M., Salami, P., Shiri, Z., and Avoli, M. (2018). Interictal oscillations and focal epileptic disorders. European Journal of Neuroscience 48, 2915-2927.

Librizzi, L., Losi, G., Marcon, I., Sessolo, M., Scalmani, P., Carmignoto, G., and Curtis, M. de (2017). Interneuronal Network Activity at the Onset of Seizure-Like Events in Entorhinal Cortex Slices. J. Neurosci. 37, 10398-10407.

Liou, J., Ma, H., Wenzel, M., Zhao, M., Baird-Daniel, E., Smith, E.H., Daniel, A., Emerson, R., Yuste, R., Schwartz, T.H., et al. (2018). Role of inhibitory control in modulating focal seizure spread. Brain 141, 2083-2097.

Magloire, V., Mercier, M.S., Kullmann, D.M., and Pavlov, I. (2018). GABAergic Interneurons in Seizures: Investigating Causality With Optogenetics. Neuroscientist 1073858418805002.

Marvin, J.S., Borghuis, B.G., Tian, L., Cichon, J., Harnett, M.T., Akerboom, J., Gordus, A., Renninger, S.L., Chen, T.-W., Bargmann, C.I., et al. (2013). An optimized fluorescent probe for visualizing glutamate neurotransmission. Nature Methods 10, 162-170.

Marvin, J.S., Scholl, B., Wilson, D.E., Podgorski, K., Kazemipour, A., Müller, J.A., Schoch, S., Quiroz, F.J.U., Rebola, N., Bao, H., et al. (2018). Stability, affinity, and chromatic variants of the glutamate sensor iGluSnFR. Nat Methods 15, 936-939.

Marvin, J.S., Shimoda, Y., Magloire, V., Leite, M., Kawashima, T., Jensen, T.P., Kolb, I., Knott, E.L., Novak, O., Podgorski, K., et al. (2019). A genetically encoded fluorescent sensor for in vivo imaging of GABA. Nat Methods 16, 763-770.

Muldoon, S.F., Villette, V., Tressard, T., Malvache, A., Reichinnek, S., Bartolomei, F., and Cossart, R. (2015). GABAergic inhibition shapes interictal dynamics in awake epileptic mice. Brain 138, 2875-2890.

Overstreet-Wadiche, L., and McBain, C.J. (2015). Neurogliaform cells in cortical circuits. Nature Reviews Neuroscience 16, 458-468.

Parrish, R.R., Codadu, N.K., Scott, C.M.-G., and Trevelyan, A.J. (2019). Feedforward inhibition ahead of ictal wavefronts is provided by both parvalbumin- and somatostatinexpressing interneurons. The Journal of Physiology 597, 2297-2314.

Pavlov, I., Kaila, K., Kullmann, D.M., and Miles, R. (2013). Cortical inhibition, pH and cell excitability in epilepsy: what are optimal targets for antiepileptic interventions? J Physiol 591, 765-774.

Prince, D.A., and Wilder, B.J. (1967). Control Mechanisms in Cortical Epileptogenic Foci*: Surround Inhibition. Arch Neurol 16, 194-202. 
Rossi, L.F., Wykes, R.C., Kullmann, D.M., and Carandini, M. (2017). Focal cortical seizures start as standing waves and propagate respecting homotopic connectivity. Nature Communications 8, 217.

Rossi, L.F., Kullmann, D.M., and Wykes, R.C. (2018). The Enlightened Brain: Novel Imaging Methods Focus on Epileptic Networks at Multiple Scales. Front Cell Neurosci 12, 82.

Schevon, C.A., Weiss, S.A., Jr, G.M., Goodman, R.R., Yuste, R., Emerson, R.G., and Trevelyan, A.J. (2012). Evidence of an inhibitory restraint of seizure activity in humans. Nat Commun 3, 1060.

Schuman, B., Machold, R.P., Hashikawa, Y., Fuzik, J., Fishell, G.J., and Rudy, B. (2019). Four Unique Interneuron Populations Reside in Neocortical Layer 1. J. Neurosci. 39, 125139.

Scimemi, A. (2014). Structure, function, and plasticity of GABA transporters. Front Cell Neurosci 8, 161.

Shuman, T., Aharoni, D., Cai, D.J., Lee, C.R., Chavlis, S., Page-Harley, L., Vetere, L.M., Feng, Y., Yang, C.Y., Mollinedo-Gajate, I., et al. (2020). Breakdown of spatial coding and interneuron synchronization in epileptic mice. Nature Neuroscience 23, 229-238.

Tremblay, R., Lee, S., and Rudy, B. (2016). GABAergic Interneurons in the Neocortex: From Cellular Properties to Circuits. Neuron 91, 260-292.

Trevelyan, A.J., Sussillo, D., Watson, B.O., and Yuste, R. (2006). Modular Propagation of Epileptiform Activity: Evidence for an Inhibitory Veto in Neocortex. Journal of Neuroscience 26, 12447-12455.

Trevelyan, A.J., Sussillo, D., and Yuste, R. (2007). Feedforward Inhibition Contributes to the Control of Epileptiform Propagation Speed. Journal of Neuroscience 27, 3383-3387.

Viitanen, T., Ruusuvuori, E., Kaila, K., and Voipio, J. (2010). The K+-Cl-cotransporter KCC2 promotes GABAergic excitation in the mature rat hippocampus. The Journal of Physiology 588, 1527-1540.

Wenzel, M., Hamm, J.P., Peterka, D.S., and Yuste, R. (2017). Reliable and Elastic Propagation of Cortical Seizures In Vivo. Cell Reports 19, 2681-2693.

Wenzel, M., Hamm, J.P., Peterka, D.S., and Yuste, R. (2019). Acute focal seizures start as local synchronizations of neuronal ensembles. J. Neurosci. 3176-18.

Yamashita, M., Kawaguchi, S., Hori, T., and Takahashi, T. (2018). Vesicular GABA Uptake Can Be Rate Limiting for Recovery of IPSCs from Synaptic Depression. Cell Reports 22, 3134-3141.

Zhang, Z.J., Koifman, J., Shin, D.S., Ye, H., Florez, C.M., Zhang, L., Valiante, T.A., and Carlen, P.L. (2012). Transition to Seizure: Ictal Discharge Is Preceded by Exhausted Presynaptic GABA Release in the Hippocampal CA3 Region. Journal of Neuroscience 32, 2499-2512. 


\section{Supplementary Figures:}

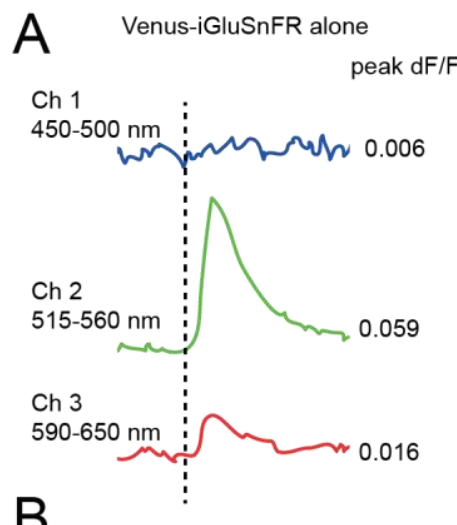

B

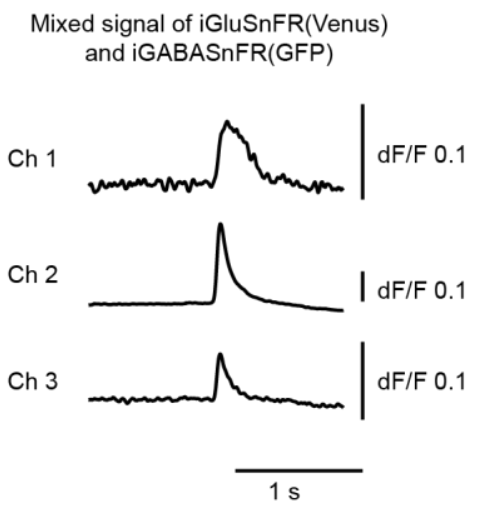

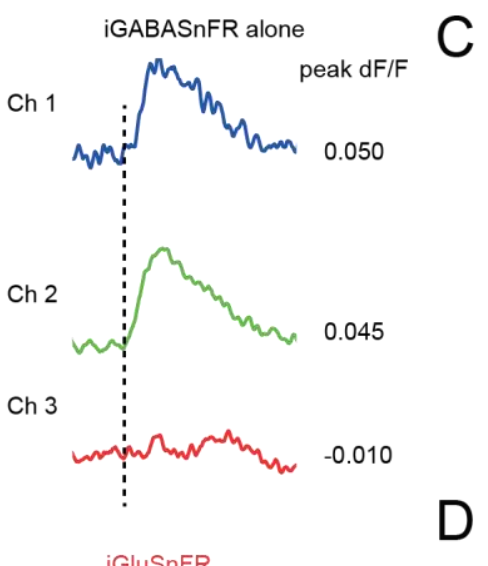
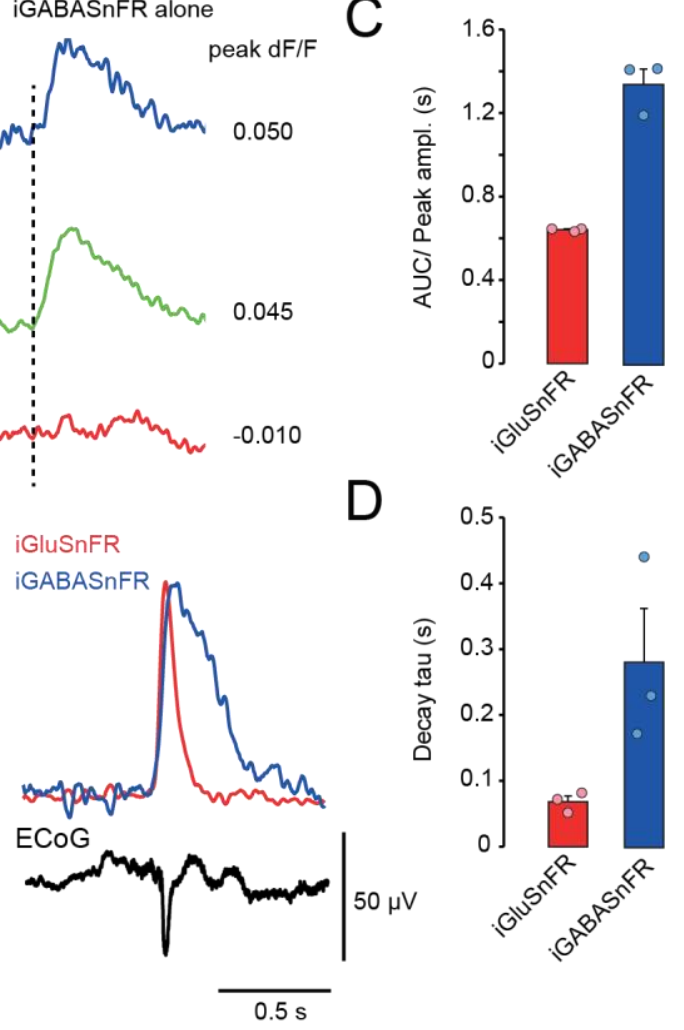

Figure 2_S1: Simultaneous imaging of iGABASnFR and iGluSnFR during interictal discharges. (A) Separate imaging of Venus-iGluSnFR (left panel) or iGABASnFR (right panel) fluorescence signal during interictal spikes via the three PMT channels, used to estimate coefficients necessary to unmix signals from both fluorophores during dual imaging. Averaged fluorescence traces (Venus-iGluSnFR, 28 events; iGABASnFR, 12 events) were aligned to IIS onset (dashed line). (B) Averaged fluorescence traces (23 events) via the three PMT channels from both sensors imaged simultaneously (left panel) and result of unmixing (right panel), together with simultaneously acquired ECoG trace. (C) Average Area Under the Curve (AUC) divided by the peak amplitude for unmixed iGABASnFR and iGluSnFR signals. (D) Same as $C$ but for the decay time constant estimated from peak to $1 \mathrm{~s}$ post-peak. Consistent with Figure 2, GABA transients were almost 3-fold longer than glutamate transients ( $n=3$ animals, Errors bars correspond to s.e.m.). 


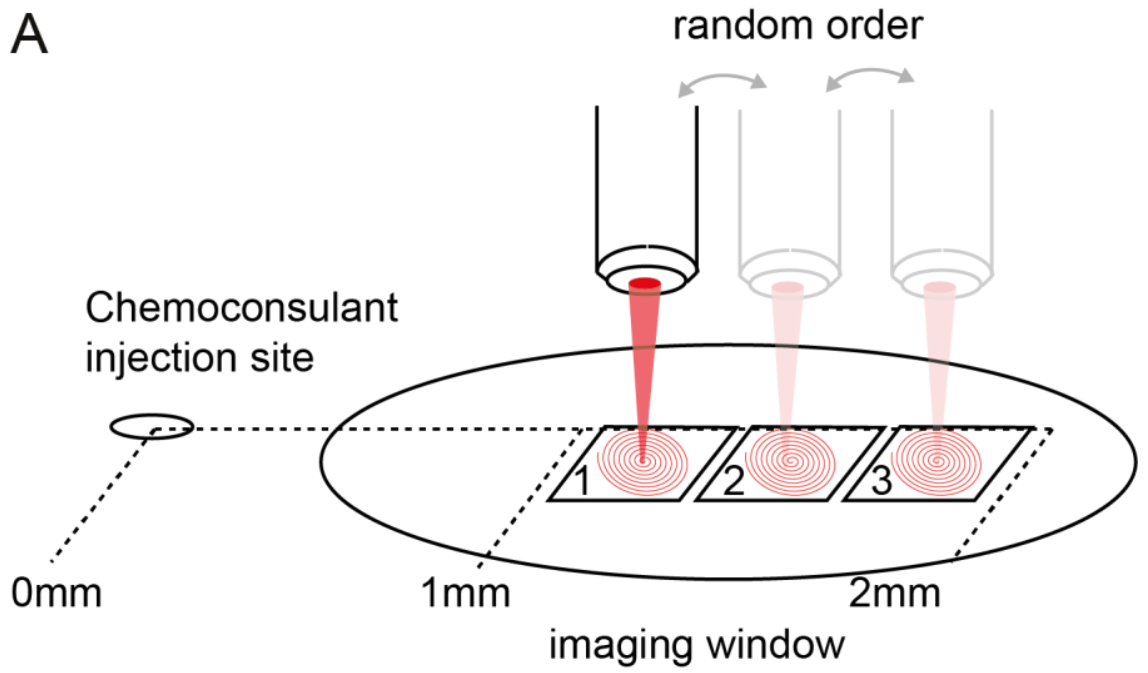

B
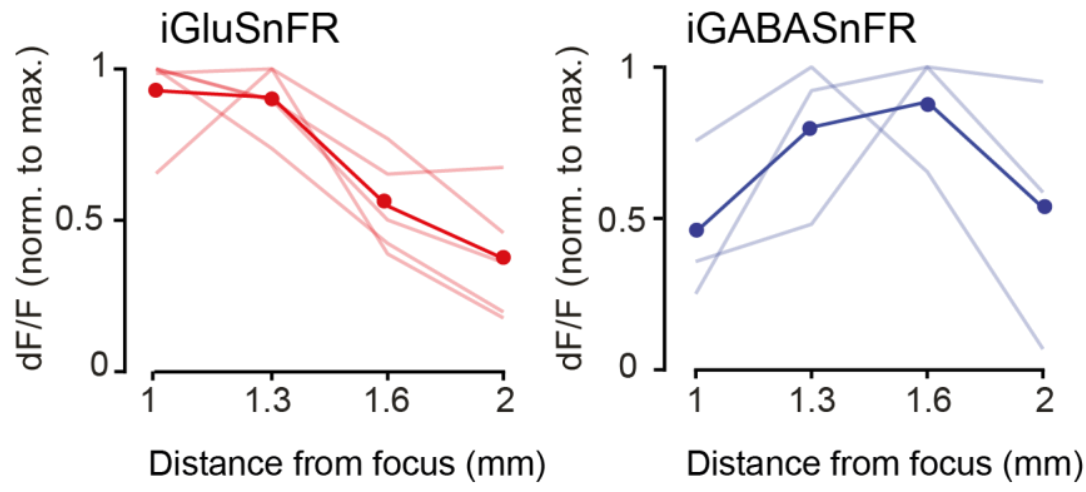

Figure 3_S1: Glutamate and GABA transients vary with distance from the focus. (A) Experimental design used to image at different locations during the interictal spiking period. At least 3 regions of interest were pre-selected before chemoconvulsant injection and then imaged in a pseudorandomized order. (B) Interictal spikes induced by injection of pilocarpine instead of picrotoxin (Figure 3). As for picrotoxin, the iGluSnFR peak transient amplitude diminished with distance from the focus, whilst the iGABASnFR transient was maximal $\sim 1.6 \mathrm{~mm}$ away (light lines: individual experiments, thick line: average). iGluSnFR: $n=5$ mice; iGABASnFR: $n=3$ mice. 
b. Vector orthogonal to the direction of propagation
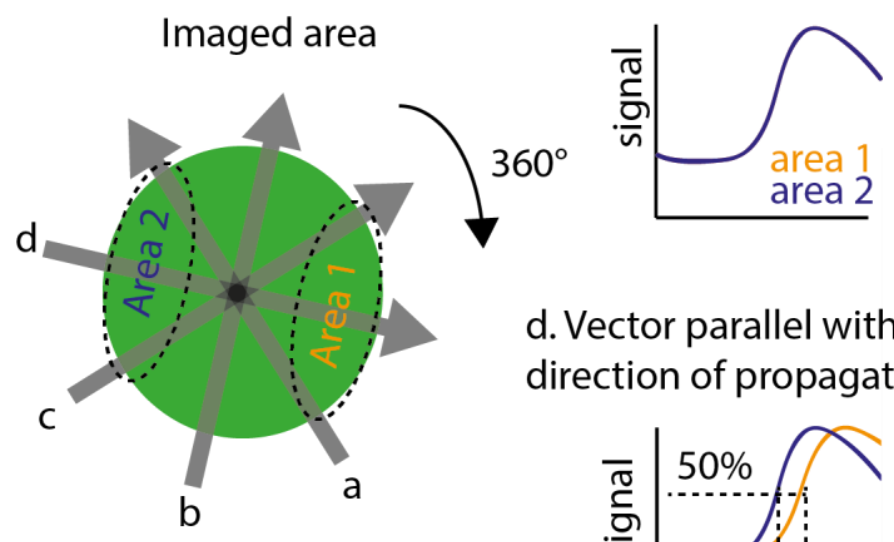

d. Vector parallel with the direction of propagation

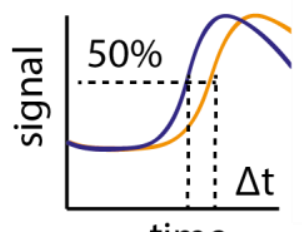

Figure 4_S1: Method to determine the direction and propagation speed of the glutamate and GABA waves during interictal discharges. 

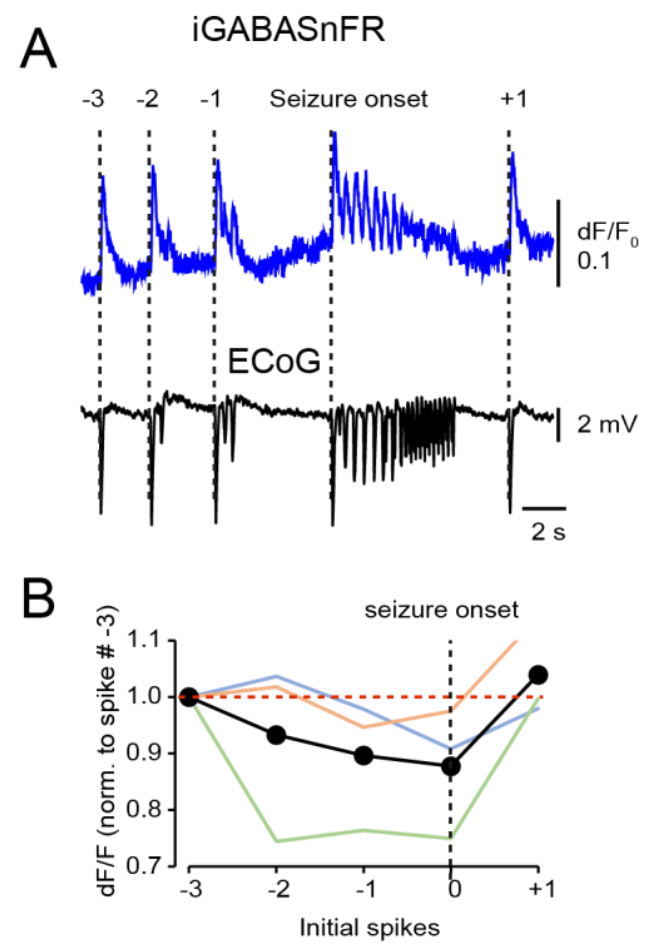

Figure 6_S1: Gradual decrease of GABA release before seizure onset. (A) Fluorescence trace replotted from Figure 5, with 3 IIDs before seizure onset, and one after, numbered. (B) Normalized fluorescence amplitude corresponding to each interictal spike and the sentinel spike, normalized to spike \# -3. (Thin coloured lines represent the mean of 3 sequences for each animal and the thick black line corresponds to the average from the 3 mice). 
A iGluSnFR

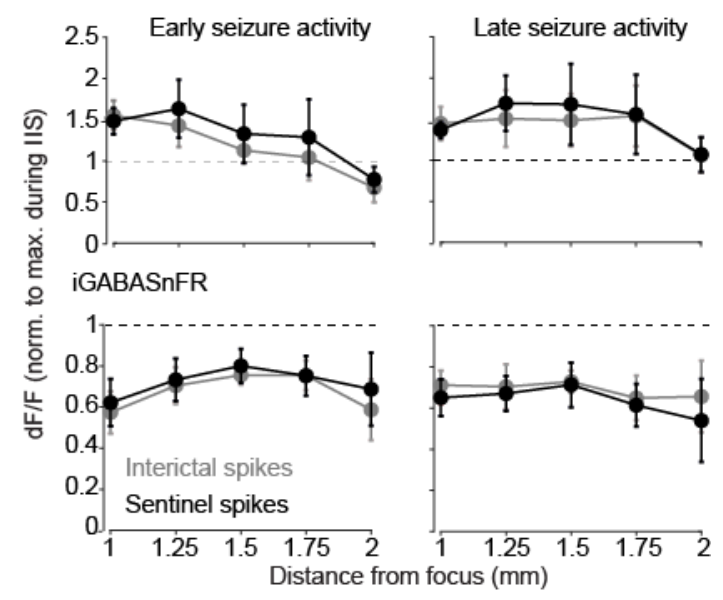

B
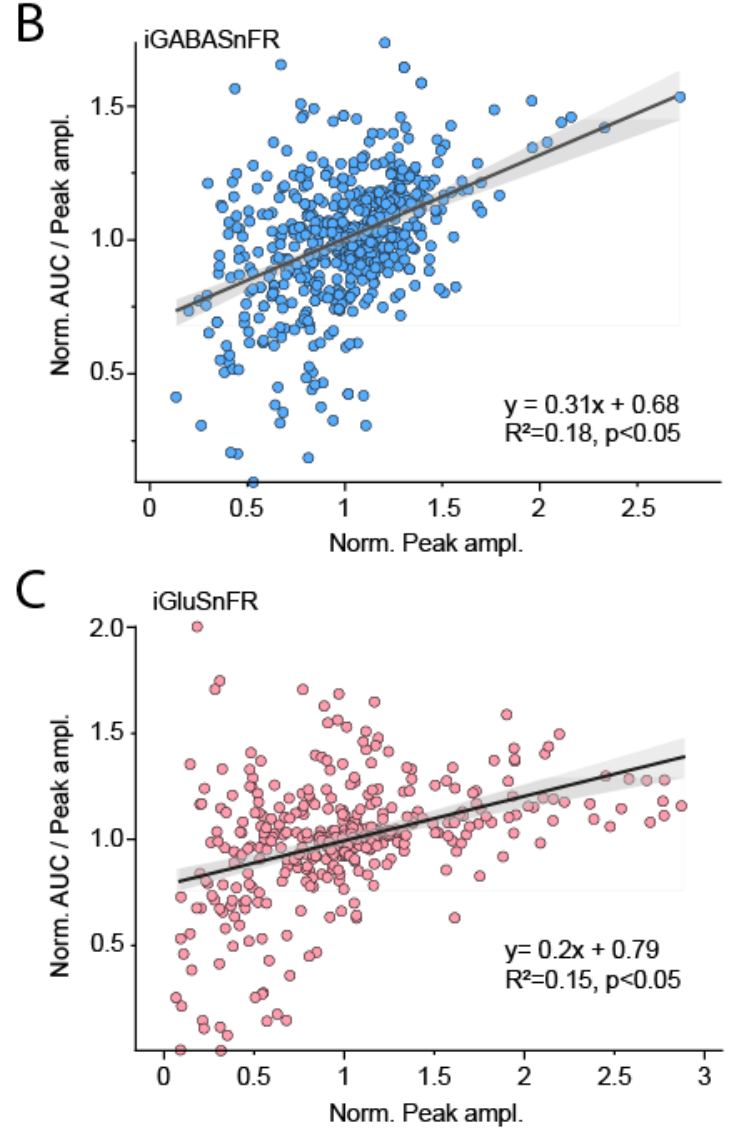

Figure 7_S1: Interictal and sentinel spikes during the early and late seizure phases.

(A) Normalized peak amplitude of iGluSnFR and iGABASnFR fluorescence transients during interictal and sentinel spikes at different distances from the focus, during ESA and LSA. Transients associated with IISs and sentinel spikes show similar spatial profiles within each phase. (B) AUC/peak amplitude plotted against peak amplitude (both normalized by the average values within each experiment) for iGABASnFR. (C) Same as B for iGluSnFR. Each point represents an individual GABA or glutamate transient during either interictal or sentinel spikes during the seizure activity period. Errors bars in (A) correspond to s.e.m.. 